\title{
Changes of liver hemodynamic and elastography parameters in patients with colorectal liver metastases receiving preoperative chemotherapy: "a note of caution"
}

Amalia Pelegrina ${ }^{1}$, Josep Martí ${ }^{1}$, Rosa Miquel ${ }^{3,4,6}$, Joana Ferrer ${ }^{1,3}$, Virginia Hernández-Gea ${ }^{2,3}$, Alba Diaz ${ }^{3,4}$, Cristina Nadal ${ }^{3,5}$, Juan Carlos García-Valdecasas ${ }^{1,3}$ and Josep Fuster ${ }^{1,3^{*}}$

\begin{abstract}
Background: New systemic chemotherapy agents have improved prognosis in patients with colorectal liver metastases (CLM), but some of them damage the liver parenchyma and ultimately increase postoperative morbidity and mortality after liver resection. The aims of our study were to determine the degree of hemodynamic and pathological liver injury in CLM patients receiving preoperative chemotherapy and to identify an association between these injuries and postoperative complications after liver resection.

Methods: This is a prospective descriptive study of patients with CLM receiving preoperative chemotherapy before curative liver resection from November 2013 to June 2014. All patients had preoperative elastography and hepatic hemodynamic evaluation. We analyzed clinical preoperative data and postoperative outcomes after grouping the patients by chemotherapy type, development of sinusoidal obstructive syndrome (SOS), and development of major complications.

Results: Eleven from the 20 patients included in the study received preoperative oxaliplatin-based chemotherapy (OBC). Nine patients had SOS at pathological analysis and five patients developed major complications. Patients receiving preoperative OBC had higher values of hepatic venous pressure gradient (HVPG) and developed more SOS and major complications. Patients developing SOS had higher values of HVPG and developed more major complications. Patients with major complications had higher values of HVPG, and patients with a HVPG of $5 \mathrm{mmHg}$ or greater had more major complications than those under $5 \mathrm{mmHg}$ (20 vs 80\%, $p=0.005)$.
\end{abstract}

Conclusions: OBC and SOS impair liver hemodynamics in CLM patients. An increase in major complications after liver resection in these patients develops at subclinical HVPG levels.

Keywords: Colorectal cancer, Liver metastases, Hepatic hemodynamic, Hepatic veins pressure gradient, Oxaliplatin, Sinusoidal obstruction syndrome

\footnotetext{
* Correspondence: jfuster@clinic.cat

'Liver Surgery and Transplant Unit, Department of Surgery, Institut de Malalties Digestives i Metabòliques Hospital Clinic Barcelona, IDIBAPS, University of Barcelona, Barcelona, Spain

${ }^{3}$ Network for Biomedical Research in Hepatic and Digestive Diseases (CIBERehd), Madrid, Spain

Full list of author information is available at the end of the article
}

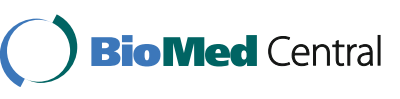

(c) The Author(s). 2017 Open Access This article is distributed under the terms of the Creative Commons Attribution 4.0 International License (http://creativecommons.org/licenses/by/4.0/), which permits unrestricted use, distribution, and reproduction in any medium, provided you give appropriate credit to the original author(s) and the source, provide a link to the Creative Commons license, and indicate if changes were made. The Creative Commons Public Domain Dedication waiver (http://creativecommons.org/publicdomain/zero/1.0/) applies to the data made available in this article, unless otherwise stated. 


\section{Background}

Colorectal carcinoma (CRC) is one of the most common cancers in the Western world, and approximately $50 \%$ of patients develop liver metastases [1]. The standard treatment for resectable colorectal liver metastases (CLM) is complete surgical resection, which offers a 5-year survival rate up to $58 \%$ [2]. In the last years, new systemic chemotherapy agents have improved the rates of complete resection and survival in patients with unresectable CLM at the time of diagnosis [3]. However, some of these agents damage the liver parenchyma and increase postoperative morbidity and mortality [4].

The main patterns of liver injury from systemic chemotherapeutic agents are chemotherapy-associated steatohepatitis and sinusoidal obstructive syndrome (SOS), which are associated with irinotecan and oxaliplatin respectively [4, 5]. Liver damage remains asymptomatic in the majority of cases, but severe oxaliplatin-induced SOS cases can evolve into non-cirrhotic portal hypertension (PHT) and ultimately complicate the safe application of subsequent therapies [4, 6]. Recently, several studies have found promising biochemical markers to diagnose the presence of sinusoidal obstruction, but these biomarkers could not quantify the degree and extent of hepatic injury nor diagnose the presence of clinical or subclinical PHT. Moreover, none of these studies measured the direct hemodynamic effects of chemotherapy-induced hepatic toxicity or quantified its related postoperative risks $[7,8]$.

Therefore, the aims of our study were to determine the hemodynamic impairment, liver stiffness, and pathological liver injury in patients with CLM who received preoperative chemotherapy and to identify a possible link between these injuries and the postoperative complications after liver resection.

\section{Methods}

This is a prospective descriptive study of patients with CLM who received preoperative chemotherapy and underwent liver resection with curative aim between November 2013 and June 2014 at our Liver Surgery and Transplantation Unit. As per the study, all patients had hepatic elastography and hemodynamic measurements to assess for liver fibrosis and hepatic venous pressure gradient (HVPG) before undergoing liver resection. We excluded patients with any pre-existing liver condition (e.g., cirrhosis, chronic liver disease) and patients with previous liver resection. The hospital ethics committee approved this study, and all included patients gave their informed consent to participate in the study when surgery for CLM was proposed.

We obtained all demographical, clinical, and pathologic data from our prospective CLM database and patients' charts. Preoperative chemotherapy (defined as received within the 6 months before CLM surgery) regimen was decided by the referring oncologist based on institutional protocols and routine practice. Patients underwent protocolized preoperative CLM staging (imaging and blood tests including tumor markers and liver function tests), postoperative care, and follow-up as previously described [9]. All patients were appointed to the Liver Surgery and Transplantation Clinic 1 month after discharge to record and grade the presence of complications during this postoperative period according to the Clavien-Dindo classification [10]. As per our Institution policy [9], CLM diagnosed before, during, or within 90 days of CRC resection were classified as synchronous and those CLM diagnosed at least 90 days after CRC resection were classified as metachronous. We defined major hepatectomy as the resection of three or more liver segments.

HVPG was obtained in all patients through a standard transjugular approach under local anesthesia. Briefly, we placed an 8-Fr venous catheter introducer in the right internal jugular vein with the Seldinger technique, measured wedge and free hepatic venous pressures, and calculated HVPG as the difference between them. We defined HVPG as normal when it was under $5 \mathrm{mmHg}$, as subclinical PHT when HVPG was between 6 and $9 \mathrm{mmHg}$, and as clinically significant PHT when HVPG was $10 \mathrm{mmHg}$ or more regardless of the presence of clinical signs of PHT [11].

Elastography was obtained in all patients lying in the dorsal decubitus position with the right arm in maximal abduction. The measures were performed on the right lobe of the liver through intercostal spaces and values expressed in kilopascals $(\mathrm{kPa})$ as previously described [12]. We defined normal elastography as stiffness under $7 \mathrm{kPa}$, presence of fibrosis when stiffness was between 7 and $14 \mathrm{kPa}$, and cirrhosis when stiffness was over $14 \mathrm{kPa}$.

Histological analysis was performed by a local experienced liver pathologist who was unaware of the treatment modality. Surgical specimens were processed and sampled as routinely according internal cut-up protocols. The number of tumors, size, distance to the transection margin, and capsular involvement were recorded, as well as the presence, distribution and approximate percentage of reticulated areas of congestion or hemorrhage, or parenchymal nodularity. At least one sample of non-tumoral liver more than $2 \mathrm{~cm}$ distant from the tumor was taken, with representation of the macroscopic lesions if present. The microscopic evaluation of the lesions on the background parenchyma was based on hematoxylin-eosin, Masson's trichrome, and reticulin stains. SOS was diagnosed and graded according to previously published criteria [13]. Briefly, the diagnosis of SOS was made when sinusoidal 
dilatation, sinusoidal congestion, and hepatocellular atrophy coexisted. Other lesions such as endothelial rupture with erythrocyte extravasation at the Disse space, central vein obliteration, nodular regenerative hyperplasia, and perisinusoidal fibrosis were also recorded. Finally, the degree of steatosis was assessed if present according to previous published criteria [14].

The patients were classified into different groups according to whether they had or not received oxaliplatin-based chemotherapy (OBC) (thus, investigated and control groups respectively), according to the presence of SOS at the pathology exam, and whether they had major postoperative complications (defined as grade III or higher in the Clavien-Dindo classification during the 30 days after the CLM operation).

All quantitative variables were tested with the Shapiro-Wilk test to ascertain normal distributions. We described quantitative variables as mean (standard deviation) or as median (interquartile range) when distribution was not normal. Qualitative variables were expressed as number and \% and compared with chi-square test. We compared groups with Student's $t$ test, Mann-Whitney $U$ test or Fisher's exact test as appropriate. A $p$ value under 0.05 was considered statistically significant. All statistical calculations were performed with the "Statistical Package for the Social Sciences" version 22.0 for Windows (SPSS, Chicago, IL, USA).

\section{Results}

\section{Patients' characteristics}

Twenty patients fulfilled the inclusion criteria during the study period. Demographic and preoperative data of the patients are shown on Tables 1 and 2. Eleven patients (55\%) received preoperative $\mathrm{OBC}$, with two of these patients receiving also biological agents (one panitumumab and another one bevacizumab). The median number of cycles of chemotherapy per patient was 6 (range 1-12). In three patients of the series, prothrombin time was under $80 \%$, and only four patients had platelets under 150,000, although all patients had platelets over 100,000. The majority of patients had normal HVPG values, and no patients had significant PHT. Three patients (15\%) had liver stiffness over $7 \mathrm{kPa}$, but no patients had stiffness values over $9 \mathrm{kPa}$.

\section{Results by chemotherapy type}

Eight $(73 \%)$ of the 11 patients receiving preoperative OBC showed SOS lesions in the non-neoplastic hepatic parenchyma. When comparing both groups, patients receiving preoperative $\mathrm{OBC}$ had significantly higher values of HVPG and higher SOS development but similar hospital stay, liver stiffness, platelets, and PT. Patients who received preoperative $\mathrm{OBC}$ had more
Table 1 Patients characteristics $(n=20)$

\begin{tabular}{|c|c|}
\hline Sex (male:female) & $13: 7$ \\
\hline Age (years) (mean $\pm S D)$ & $63.35 \pm 11.49$ \\
\hline Body mass index ( $\left.\mathrm{kg} / \mathrm{m}^{2}\right)$ (median, range) & $25(22.9-29.5)$ \\
\hline \multicolumn{2}{|l|}{ ASA grade $(n, \%)$} \\
\hline$|-| \mid$ & $12(60 \%)$ \\
\hline III-IV & $8(40 \%)$ \\
\hline Platelets (mean \pm SD) & $208,600 \pm 73,650$ \\
\hline Prothrombin activity percentage (mean $\pm \mathrm{SD}$ ) & $94.47 \pm 10.44$ \\
\hline HVPG $(m m H g)($ mean $\pm S D)$ & $3.80 \pm 1.67$ \\
\hline Elastography (kPa) (mean $\pm \mathrm{SD})$ & $5.41 \pm 1.67$ \\
\hline No. of metastases (mean \pm SD) & $2.26 \pm 1.94$ \\
\hline \multicolumn{2}{|l|}{ Occurrence of metastases ( $n, \%)$} \\
\hline Synchronous & $9(45 \%)$ \\
\hline Metachronous & $11(55 \%)$ \\
\hline Steatosis (\%) & 45 \\
\hline Inflammation (\%) & 45 \\
\hline Fibrosis (\%) & 65 \\
\hline
\end{tabular}

ASA American Society of Anesthesiologists, HVPG hepatic venous pressure gradient

major complications than the ones not receiving OBC, with all five patients with major complications receiving OBC (Table 3).

\section{Histological findings}

Nearly $50 \%$ of overall patients in our series presented with histological abnormalities leading to the diagnosis of SOS, all of them having received preoperative OBC. Patients with SOS had higher values of HVPG and had received more chemotherapy cycles, but they had similar liver stiffness, platelets, PT, length of hospital stay, and major complications than those patients without SOS (Table 4).

\section{Analysis of complications}

Median length of hospital stay was 7 days (range 6-10), and overall complication rate was $70 \%$. The majority of the major complications were basically general (intraabdominal collections, pleural effusions), although two of them were related to biliary leaks that required reoperation However, the majority of patients' complications (64\%) were graded as I-II, and there were no patients with IV or higher grade complications. Four $(80 \%)$ of the five patients who presented with major complications had SOS on the pathologic analysis. Patients with complications had similar values of HVPG, PT, liver stiffness, platelets, and length of hospital stay than the patients without complications (data not shown). However, patients with major complications had higher values of HVPG and lower PT values but similar liver 
Table 2 Hemodynamic measurements, elastography, laboratory findings, chemotherapy, SOS, and complications

\begin{tabular}{|c|c|c|c|c|c|c|c|c|}
\hline Patient & HVPG & Elastography & Platelets & PT (\%) & Chemotherapy (cycles) & OBC & SOS & Complications \\
\hline 1 & 4.50 & 6.80 & 336,000 & 100 & FOLFOX [6] & Yes & Yes & 0 \\
\hline 2 & 6.50 & N/A & 157,000 & 100 & FOLFOX [8] & Yes & Yes & । \\
\hline 3 & 2.00 & N/A & 135,000 & 76 & Capecitabine [2] & - & - & 0 \\
\hline 4 & 4.00 & N/A & 105,000 & 98 & FOLFOX [9] & - & Yes & I \\
\hline 5 & 7.00 & 7.90 & 150,000 & 95 & FOLFOXIRI [8] & Yes & Yes & $\| \mathrm{II}$ \\
\hline 6 & 6.00 & 8.40 & 136,000 & 89 & FOLFOX [6] & Yes & Yes & $\| \mathrm{A}$ \\
\hline 7 & 2.00 & N/A & 196,000 & 99 & FOLFOX + FOLFIRI [12] & - & - & I \\
\hline 8 & 2.50 & 3.80 & 192,000 & 100 & FOLFOX [4] & Yes & - & 1 \\
\hline 9 & 2.50 & 4.70 & 199,000 & 100 & Capecitabine [1] & - & - & 0 \\
\hline 10 & 1.50 & N/A & 197,000 & 100 & FOLFOX + bevacizumab [7] & - & - & 1 \\
\hline 11 & 5.00 & N/A & 191,000 & 100 & FOLFOX [4] & Yes & Yes & $\| I I B$ \\
\hline 12 & 2.50 & 5.20 & 274,000 & 100 & Capecitabine [6] & - & - & 0 \\
\hline 13 & 4.00 & 4.40 & 157,000 & 100 & FOLFOX [5] & Yes & Yes & $\| B$ \\
\hline 14 & 4.00 & 6.10 & 135,000 & 100 & FOLFOX [3] & - & - & । \\
\hline 15 & 4.00 & 7.60 & 283,000 & 100 & FOLFOX [6] & Yes & - & 1 \\
\hline 16 & 2.50 & 3.60 & 215,000 & 92 & FOLFIRI + cetuximab [6] & - & - & । \\
\hline 17 & 6.00 & 4.50 & 291,000 & 78 & TOMOX [6] & Yes & Yes & $\| I A$ \\
\hline 18 & 2.00 & 5.20 & 157,000 & 100 & FOLFOX + TOMOX + panitumumab [8] & - & - & 0 \\
\hline 19 & 4.5 & 4.00 & 331,000 & 100 & FOLFOX [5] & Yes & Yes & 0 \\
\hline 20 & 3 & 3.50 & 335,000 & 62.50 & XELOX [5] & Yes & - & $\| \mathrm{II}$ \\
\hline
\end{tabular}

N/A not available

stiffness, platelets, and length of hospital stay than the patients without major complications (Table 5). Also, major resections were not a factor that was statistically associated with more complications (although nearly significant), age, or comorbidities (defined by the ASA grade). When performing a ROC curve, $4.25 \mathrm{mmHg}$ was the HVPG value that gave better sensitivity and specificity ( 80 and $80 \%$ respectively) for major complications, and the AUC was $0.867(p=0.016)$. In this direction, patients with a HVPG of $5 \mathrm{mmHg}$ or greater had more major complications than those under $5 \mathrm{mmHg}$ (20 vs $80 \%, p=0.005)$.

\section{Discussion}

The appearance of new systemic chemotherapy agents has increased resectability and survival rates in patients with CLM, but some of these agents damage the liver parenchyma and increase postoperative morbidity and mortality $[4,5,15]$. One of the most well-known liver injury patterns is oxaliplatin-induced SOS, which can evolve into non-cirrhotic PHT in serious cases [6]. However, no studies have shown the hemodynamic effects of chemotherapy-induced hepatic toxicity by direct measurement of the vascular pressures by transjugular hemodynamic procedure of the liver, or quantified the

Table 3 Comparison of patients with and without oxaliplatin-based chemotherapy

\begin{tabular}{llll}
\hline & $\begin{array}{l}\text { Chemotherapy without oxaliplatin } \\
(n=9)\end{array}$ & $\begin{array}{l}\text { Oxaliplatin-based chemotherapy } \\
(n=11)\end{array}$ & $p$ value \\
\hline HVPG $(\mathrm{mmHg})^{\mathrm{a}}$ mean (SD) & $2.56(.88)$ & $4.82(1.44)$ & 0.001 \\
Elastography (kPa) ${ }^{\mathrm{a}}$ mean (SD) & $4.96(.91)$ & $5.66(1.98)$ & 0.478 \\
Platelets (log) $)^{\mathrm{a}}$ mean (SD) & $5.24(.13)$ & $5.34(.16)$ & 0.127 \\
PT (\%) $)^{\mathrm{b}}$ median; (IQR) & $100.0(98.0,100.0)$ & $100(89.0,100.0)$ & 0.998 \\
Length of hospital stay (days) $^{\mathrm{b}}$ Median (IQR) & $7.0(5.0,9.0)$ & $8.0(7.0,10.0)$ & 0.412 \\
SOS development $n(\%)^{\mathrm{c}}$ & $1(11.1)$ & $8(72.7)$ & 0.010 \\
Major complications $n(\%)^{\mathrm{c}}$ & $0(0)$ & $5(45.5)$ & 0.038 \\
\hline
\end{tabular}

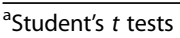

${ }^{b}$ Mann-Whitney $U$-test

'Fisher's exact test 
Table 4 Comparison of patients with and without SOS

\begin{tabular}{|c|c|c|c|}
\hline & No SOS $(n=11)$ & $\operatorname{SOS}(n=9)$ & $p$ value \\
\hline HVPG $(\mathrm{mmHg})^{\mathrm{a}}$ mean $(\mathrm{SD})$ & $2.59(.80)$ & $5.28(1.12)$ & $<0.001$ \\
\hline Elastography $(\mathrm{kPa})^{\mathrm{a}}$ mean (SD) & $4.96(1.40)$ & $6.00(1.94)$ & 0.266 \\
\hline Platelets (log) ${ }^{a}$ mean (SD) & $5.31(0.13)$ & $5.28(0.18)$ & 0.698 \\
\hline PT $(\%)^{\mathrm{b}}$ median (IQR) & $100(92.0,100)$ & $100(95.0,100)$ & 0.882 \\
\hline Length of hospital stay (days) $)^{\mathrm{b}}$ median (IQR) & $7.0(5.0,12.0)$ & $8.0(7.0,8.0)$ & 0.998 \\
\hline Major complications $n(\%)^{c}$ & $1(9.1)$ & $4(44.4)$ & 0.127 \\
\hline
\end{tabular}

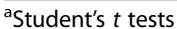

${ }^{\mathrm{b}}$ Mann-Whitney U-test

'Fisher's exact test

association between these hemodynamic effects and postoperative complications $[7,8]$.

Oxaliplatin is a systemic chemotherapy agent that prevents cell division and replication by crosslinking DNA strands. In the liver, oxaliplatin damages mainly the sinusoidal endothelial cells and causes SOS, which ultimately decreases the hepatic functional reserve and increases the risk of bleeding during surgery [4, 5]. Several studies have intended to predict SOS based on blood and serum parameters like platelet count, aspartate aminotransferase, gamma glutamyl transferase, alkaline phosphatase, and aspartate aminotransferase to platelet ratio index. However, these biomarkers have not been proved useful to quantify the degree and extent of hepatic injury, and still no large studies have validated them as a reliable indicator of sinusoidal damage $[7,8]$. On the other hand, there are no studies focused on the presence of clinical or subclinical PHT induced by OBC, something that only direct measurement of liver hemodynamic parameters can accurately diagnose [11]. Our study showed that patients receiving preoperative OBC had higher values of HVPG when compared to patients not receiving $\mathrm{OBC}$ and also that SOS development was associated with receiving $\mathrm{OBC}$ (especially when the number of chemotherapy cycles increased). This latter observation is consistent with previous studies showing a clear link between $\mathrm{OBC}$ and the development of SOS $[4,6,16,17]$. On the other hand, the increase of HVPG in patients receiving OBC or developing $\mathrm{SOS}$ clearly shows that $\mathrm{OBC}$ impairs liver pathology and hemodynamic parameters. This observation favors the logical and already postulated pathogenical hypothesis that preoperative $\mathrm{OBC}$ would lead to sinusoidal obstruction and increased intrahepatic resistance and would subsequently increase HVPG [6, 17].

Several studies have highlighted the increase of postoperative morbidity and mortality in CLM patients treated with chemotherapy drugs and the important role of a proper operative timing given the frequent attenuation of liver injury after stopping the systemic chemotherapy agent [18-20]. The ideal situation would be to predict the increased postoperative risk before surgery by diagnosing SOS in patients treated with OBC. To this objective, some authors have proposed to perform preoperative percutaneous or transjugular liver biopsies or even a diagnostic laparoscopy to rule out significant liver alterations and select the best possible therapeutic strategy for each patient $[5,18,19]$. However, as SOS histological lesions may be focal or even patchy, these alternatives remain controversial because of the risks of sampling error and subsequent underdiagnosis of sinusoidal lesions. Lately, Nakano et al. have identified several preoperative factors associated to sinusoidal damage (6 or more cycles of oxaliplatin, abnormal preoperative liver function tests, increased indocyanine green retention rate, and female sex) that can help to get the diagnosis in the preoperative setting [20]. In our study, however, we could not find differences in the preoperative biochemical parameters between patients developing SOS or not, nor did we find differences in

Table 5 Comparison according to the presence of major complications

\begin{tabular}{llll}
\hline & Absent or minor complications $(n=15)$ & Major complications $(n=5)$ & $p$ value \\
\hline HVPG $(\mathrm{mmHg})^{\mathrm{a}}$ mean (SD) & $3.27(1.36)$ & $5.40(1.52)$ & 0.008 \\
${\text { Elastography }(\mathrm{kPa})^{\mathrm{a}} \text { mean (SD) }}$ & $5.14(1.33)$ & $6.08(2.44)$ & 0.511 \\
Platelets $(\mathrm{log})^{\mathrm{a}}$ mean (SD) & $5.29(0.15)$ & $5.32(0.17)$ & 0.772 \\
PT (\%) $)^{\mathrm{b}}$ median (IQR) & $100(99.0 .100 .0)$ & $89.0(78.0,95)$ & 0.033 \\
Length of hospital stay (days) $^{\mathrm{b}}$ median (IQR) & $7.0(6.0,9.0)$ & $8.5(7.0,10.0)$ & 0.445 \\
\hline
\end{tabular}

astudent's $t$ tests

${ }^{\mathrm{b}}$ Mann-Whitney U-test 
the major complication rate between patients developing SOS. Nevertheless, the specific aim of our study and the limited number of patients included in it can easily explain these observations because we designed our study towards successfully finding differences in hemodynamic alterations but not towards finding differences in the preoperative biochemical parameters or in major complications. Despite this limitation, that adds to not being able to prove that increases in HVPG are the main cause for postoperative complications, we believe that the large amount of existing studies proving the relation between SOS development and the subsequent alteration of preoperative biochemical parameters (with increased complications) makes it unnecessary to challenge this well-studied fact and still keeps the validity of our study.

PHT becomes clinically significant when HVPG rises above $10 \mathrm{mmHg}$ and the patient develops cirrhotic complications like variceal bleeding, ascites, or thrombocytopenia [11]. Moreover, several studies on patients with liver cirrhosis undergoing liver resection and other surgical procedures have demonstrated the importance of PHT. In this direction, more than $50 \%$ of cirrhotic patients classified as Child-Pugh A will decompensate after surgery, with subsequent decreased quality of life and long-term survival [21-23]. Although splenomegaly and thrombocytopenia are usually indirect clinical signs of PHT in cirrhosis or in patients receiving OBC [6-8], direct hepatic hemodynamic evaluation is currently the best method to study and diagnose PHT $[11,24]$. To the present one, no study had directly measured the liver hemodynamic effects of chemotherapy-induced liver damage or attempted to correlate their hemodynamic changes with the development of complications. In our study, patients with major complications had higher HVPG than those not developing major complications, and major complications developed at lower cutoff HVPG levels than the usually accepted to define clinically significant PHT in patients with chronic liver diseases [11, 24]. These interesting new findings suggest a causal association between OBCinduced liver hemodynamic impairment and major complications but also show that the development of major complications in CLM patients receiving preoperative OBC starts at earlier HVPG levels than those universally accepted as clinically relevant in patients with chronic liver diseases. Although the design of our study cannot elucidate the cause for this earlier manifestation of initial hemodynamic impairment, we hypothesize that OBCrelated liver injury would cause significant liver damage and an increase in major postoperative complications because of the lack of compensatory mechanisms against the hemodynamic disturbances that only develop in patients with chronic liver diseases. More important, however, it is the fact that clinicians taking care of CLM patients receiving $\mathrm{OBC}$ should be aware of the development of this early significant hemodynamic impairment and specifically look for it in advance because of its severe clinical consequences.

Elastography has been advocated to be a useful tool to diagnose the degree of liver fibrosis and PHT through liver stiffness measurement, although several factors including sex, liver inflammation, cholestasis, hepatocellular carcinoma, liver congestion, and disease etiology may influence its results [12, 25-27]. One of the few studies about liver elastography in patients with CRC has shown that liver stiffness increases within $48 \mathrm{~h}$ after starting $\mathrm{OBC}$ and normalizes within 2 weeks after treatment in most cases [28]. In our study, we could not find any difference in liver stiffness when comparing by whether the patient had received OBC, developed SOS or suffered major complications. The most likely reason for this lack of association is that, as observed in other studies, elastography results only correlate with those obtained by hepatic hemodynamic evaluation for HVPG above $10 \mathrm{mmHg}[12,25-27]$. Also, elastography results are mainly affected by the amount of liver fibrosis but not by the degree of sinusoidal obstruction, a fact that is better studied with direct liver hemodynamic measurements [11, 29]. We believe that in order to find some differences in liver stiffness, our study should have included patients with higher hemodynamic impairment, something that was not done because of the exploratory nature of our study. However, this limitation needs to be taken into account when designing further studies specifically aiming to find the value of elastography to predict chemotherapy-induced liver injury.

Even though this is the first study to directly analyze liver hemodynamic parameters in CLM patients receiving preoperative chemotherapy, we acknowledge that our study had some limitations. Small sample size is probably the most obvious and important one, but this limitation comes from the study being necessarily an exploratory analysis because no similar studies existed and because potential complications that can derive from direct hepatic hemodynamic measurement advised against a large preliminary study [29]. Nevertheless, we feel that this is a minor limitation because sample size accounted for not finding differences in some variables that have extensively described by other studies but that were not the primary objective of our study. In this sense, future similar studies should aim to enroll more patients and include more heterogeneous patients (e.g., patients with previous chronic liver diseases or with longer chemotherapy treatments) in order to enable stratification and ascertain the effect of other preoperative characteristics in the postoperative results. 
Other limitations that can be found in our study include the concern for overdiagnosis of SOS after OBC, which derives from the fact that nearly $75 \%$ of patients receiving $\mathrm{OBC}$ were histologically diagnosed with SOS, and also the fact that HVPG measurement was done only after receiving chemotherapy, raising the concern that maybe there were some prior alterations in HVPG before chemotherapy. We feel that further studies with different designs adapted to these limitations can help to solve these questions that our current design study cannot answer.

\section{Conclusions}

To conclude, our preliminary study confirmed the association between OBC and SOS in CLM patients and showed that OBC-induced SOS impairs liver hemodynamics, moreover describing that major complications in these patients start to develop at lower HVPG levels than those universally accepted as clinically relevant in patients with chronic liver diseases. These interesting results should encourage further analyses to define the effect of other preoperative characteristics in CLM patients receiving $\mathrm{OBC}$ and thus improve postoperative results.

\section{Abbreviations}

CLM: Colorectal liver metastases; CRC: Colorectal carcinoma; HVPG: Hepatic venous pressure gradient; OBC: Oxaliplatin-based chemotherapy; PHT: Portal hypertension; SOS: Sinusoidal obstructive syndrome

\section{Acknowledgements}

Not applicable

\section{Funding}

The Association Llavaneres contra el Cáncer supported the work of Josep Fuster.

\section{Availability of data and materials}

Data supporting our finding can be accessed upon request to the corresponding author.

\section{Authors' contributions \\ AP participated in the design of the study, performed the statistical analysis, and drafted the manuscript. JM conceived the study, performed the statistical analysis, and drafted the manuscript. RM participated in the design of the study, carried the histological analysis, and helped to draft the manuscript. JF participated in the design of the study and helped to draft the manuscript. VHG performed the measurement of liver hemodynamic parameters and helped to draft the manuscript. AD carried the histological analysis and helped to draft the manuscript. CN participated in the design and coordination of the study and helped to draft the manuscript. JGV participated in the design and coordination of the study. JF conceived the study, participated in its design and coordination, and helped to draft the manuscript. All authors read and approved the final manuscript.}

\section{Ethics approval and consent to participate}

The study obtained the approval from the Ethics Committee at our Institutional Review Board for Clinical Research. All patients gave their informed consent before being scheduled for measurement of liver hemodynamic parameters and liver resection.

\section{Consent for publication}

Not applicable

\section{Competing interests}

The authors declare that they have no competing interests.

\section{Publisher's Note}

Springer Nature remains neutral with regard to jurisdictional claims in published maps and institutional affiliations.

\section{Author details}

${ }^{1}$ Liver Surgery and Transplant Unit, Department of Surgery, Institut de Malalties Digestives i Metabòliques Hospital Clinic Barcelona, IDIBAPS, University of Barcelona, Barcelona, Spain. ${ }^{2}$ Barcelona Hemodynamics Laboratory, Liver Unit, IDIBAPS, CIBERehd, Hospital Clínic, University of Barcelona, Barcelona, Spain. ${ }^{3}$ Network for Biomedical Research in Hepatic and Digestive Diseases (CIBERehd), Madrid, Spain. ${ }^{4}$ Department of Pathology, Hospital Clinic Barcelona, IDIBAPS, University of Barcelona, Barcelona, Spain. ${ }^{5}$ Institut de Malalties Hemato-Oncológiques Hospital Clinic Barcelona, IDIBAPS, University of Barcelona, Barcelona, Spain. ${ }^{6}$ Institute of Liver Studies King's College Hospital, Denmark Hill SE5 9RS, UK.

Received: 22 August 2017 Accepted: 5 December 2017

Published online: 16 December 2017

\section{References}

1. Torre LA, Bray F, Siegel RL, Ferlay J, Lortet-Tieulent J, Jemal A. Global cancer statistics, 2012. CA Cancer J Clin. 2015;65:87-108.

2. Veereman $G$, Robays J, Verleye L, Leroy R, Rolfo C, et al. Poole analysis of the surgical treatment for colorectal cancer liver metastases. Crit Rev Oncol Hematol. 2015;94(1):122-35.

3. Gustavsson B, Carlsson G, Machover D, Petrelli N, Roth A, et al. A review of the evolution of systemic chemotherapy in the management of colorectal cancer. Clin Colorectal Cancer. 2015;14(1):1-10.

4. Zorzi D, Laurent A, Pawlik TM, Lauwers GY, Vauthey JN, Abdalla EK. Chemotherapy-associated hepatotoxicity and surgery for colorectal liver metastases. Br J Surg. 2007:94(3):274-86.

5. Chun YS, Laurent A, Maru D, Vauthey JN. Management of chemotherapyassociated hepatotoxicity in colorectal liver metastases. Lancet Oncol. 2009; 10(3):278-86.

6. Slade $\mathrm{JH}$, Alattar ML, Fogelman DR, Overman MJ, Agarwal A, Maru DM, et al. Portal hypertension associated with oxaliplatin administration: clinical manifestations of hepatic sinusoidal injury. Clin Colorectal Cancer. 2009:8(4): 225-30

7. Soubrane $\mathrm{O}$, Brouquet A, Zalinski S, Terris B, Brézault C, Mallet V, et al. Predicting high grade lesions of sinusoidal obstruction syndrome related to oxaliplatin-based chemotherapy for colorectal liver metastases. Ann Surg. 2010;251(3):454-60.

8. Overman MJ, Maru DM, Charnsangavej C, Loyer EM, Wang H, Pathak P, et al. Oxaliplatin-mediated increase in spleen size as a biomarker for the development of hepatic sinusoidal injury. J Clin Oncol. 2010;28(15):2549-55.

9. Martí J, Modolo M, Fuster MJ, Comas J, Cosa R, et al. Prognostic factors and time-related changes influence results of colorectal liver metastases surgical treatment: a single-center analysis. World J Gastroenterol. 2009; 15(21):2587-94

10. Dindo D, Demartines N, Clavien PA. Classification of surgical complications. A new proposal with evaluation in a cohort of 6336 patients and results of a survey. Ann Surg. 2004;240:205-13.

11. Bosch J, Abraldes JG, Berzigotti A, García-Pagan JC. The clinical use of HVPG measurements in chronic liver disease. Nat Rev Gastroenterol Hepatol. 2009; 6(10):573-82.

12. Berzigotti A, Seijo S, Arena U, Abraldes JG, Vizzutti F, García-Pagán JC, et al. Elastography, spleen size and platelet count identify portal hypertension in patients with compensated cirrhosis. Gastroenterology. 2013;144:102-11.

13. Rubbia-Brandt L, Audard V, Sartoretti P, Roth AD, Brezault C, et al. Severe hepatic sinusoidal obstruction associated with oxaliplatin based chemotherapy in patients with metastatic colorectal cancer. Ann Oncol 2004; 15(3): 406-6.

14. Brunt EM, Janney CG, Di Bisceglie AM, Neuschwander-Tetri BA, Bacon BR. Nonalcoholic steatohepatitis: a proposal for grading and staging the histological lesions. Am J Gastroenterol. 1999;94:2467-7244.

15. Robinson SM, Wilson CH, Burt AD, Manas DM, White SA. Chemotherapyassociated liver injury in patients with colorectal liver metastases: a systematic review and meta-analysis. Ann Surg Oncol. 2012;19(13):4287-99.

16. Aloia T, Sebagh M, Plasse M, Karam V, Lévi F, Giacchetti S, et al. Liver histology and surgical outcomes after preoperative chemotherapy with 
fluorouracil plus oxaliplatin in colorectal cancer liver metastases. J Clin Oncol. 2006;24(31):4983-90.

17. Hubert C, Sempoux C, Humblet Y, van den Eynde M, Zech F, Leclercq I, Gigot JF. Sinusoidal obstruction syndrome (SOS) related to chemotherapy for colorectal liver metastases: factors predictive of severe SOS lesions and protective effect of bevacizumab. HPB. 2013;15:858-64.

18. Khan AZ, Morris-Stiff G, Makuuchi M. Patterns of chemotherapy-induced hepatic injury and their implications for patients undergoing liver resection for colorectal liver metastases. J Hepato-Biliary-Pancreat Surg. 2009;16(2): 137-44.

19. Viganò L, Capussotti L, De Rosa G, De Saussure WO, Mentha G, RubbiaBrandt L. Liver resection for colorectal metastases after chemotherapy. Impact of chemotherapy-related liver injuries, pathological tumor response, and micrometastases on long-term survival. Ann Surg. 2013;258(5):731-40.

20. Nakano H, Oussoultzoglou E, Rosso E, Casnedi S, Chenard-Neu MP, Dufour $P$, et al. Sinusoidal injury increases morbidity after major hepatectomy in patients with colorectal liver metastases receiving preoperative chemotheraphy. Ann Surg. 2008;247(1):118-24.

21. Goede B, Klitsie PJ, Lange JF, Metselaar HJ, Kazemier G. Morbidity and mortality related to non-hepatic surgery in patients with liver cirrhosis: a systematic review. Best Pract Res Clin Gastroenterol. 2012;26(1):47-59.

22. Im GY, Lubezky N, Facciuto ME, Schiano TD. Surgery in patients with portal hypertension: a preoperative checklist and strategies for attenuating risk. Clin Liver Dis. 2014;18(2):477-505.

23. Bruix J, Castells A, Bosch J, Feu F, Fuster J, Garcia-Pagan JC, Visa J, et al. Surgical resection of hepatocellular carcinoma in cirrhotic patients: prognostic value of preoperative portal pressure. Gastroenterology. 1996; 111(4):1018-22.

24. Abraldes JG, Sarlieve P, Tandon P. Measurement of portal pressure. Clin Liver Dis. 2014;18(4):779-92.

25. Ferraioli G, Tinelli C, Dal Bello B, Zicchetti M. Performance of liver stiffness measurements by transient elastography in chronic hepatitis. World J Gastroenterol. 2013;19(1):49-56.

26. Friedrich-Rust M, Poynard T, Castera L. Critical comparison of elastography methods to assess chronic liver disease. Nat Rev Gastroenterol Hepatol. 2016; [Epub ahead of print]

27. Chang PE, Goh GB, Ngu JH, Tan HK, Tan CK. Clinical applications, limitations and future role of transient elastography in the management of liver disease. World J Gastrointest Pharmacol Ther. 2016;7(1):91-106.

28. Oki E, Kakeji Y, Taketomi A, Yamashita Y, Ohgaki K, Harada N, Iguchi T, et al. Transient elastography for the prediction of oxaliplatin-associated liver injury in colon cancer patients: a preliminary analysis. J Gastrointest Cancer. 2008; 39(1-4):82-5.

29. Kumar A, Sharma P, Sarin SK. Hepatic venous pressure gradient measurement: time to learn. Indian J Gastroenterol. 2008;27(2):74-80.

\section{Submit your next manuscript to BioMed Central and we will help you at every step:}

- We accept pre-submission inquiries

- Our selector tool helps you to find the most relevant journal

- We provide round the clock customer support

- Convenient online submission

- Thorough peer review

- Inclusion in PubMed and all major indexing services

- Maximum visibility for your research

Submit your manuscript at www.biomedcentral.com/submit

C) Biomed Central 